\title{
3-dimensional Morphologies of Truncated Ferrite Nanoparticles
}

\author{
R. Swaminathan ${ }^{*}$, N.T. Nuhfer ${ }^{*}$ and M.E. McHenry ${ }^{*}$ \\ *Materials Science and Engineering, Carnegie Mellon University, Pittsburgh, PA-15213
}

In nanoparticles, there is an increased fraction of atoms at the surface that are not fully coordinated and therefore contribute differently to properties than do atoms in the bulk material. The justification for the relative importance of surfaces in nanoparticles must be naturally extended to consider the crystallography of the surfaces present. NiZn ferrite nanoparticles synthesized using an RF plasma torch [1], exhibit cuboctahedral morphologies with exclusively (100) and (111) type surfaces. These surfaces contribute differently [2] to surface magnetic anisotropy, which determines the high frequency magnetic response in these materials. In this paper, we explore the 3-dimensional morphologies of the ferrite nanoparticles using electron tomographic techniques.

The 2-dimensional TEM projection images indicated that the small particles $(<20 \mathrm{~nm})$ were octahedral (only (111) surfaces) while the larger particles $(20-50 \mathrm{~nm})$ were truncated octahedral (having both (100) and (111) surfaces) in shape. To confirm the 3-dimensional nanoparticle morphologies, tilt experiments were performed on selected areas of the sample using a TECNAI FEG TEM (F20 model) with a single-tilt specimen holder. A series of $2 \mathrm{D}$ tilt images (at $2^{\circ}$ tilt intervals) over a tilt range of $\pm 45^{\circ}$ (with respect to the primary electron beam) were recorded using the attached CCD camera. Image shifts and focus changes were performed for all tilt angles to always keep the specimen in the field of view in proper focus. The ferrite nanoparticles were quite stable to electron exposure over long time periods, and hence, electron irradiation damage to the sample is not a critical issue while obtaining a series of around 45 images in a specific area. The recorded series of 2D images was manually aligned, stacked and then rotated in the corresponding tilt axis to obtain the 3D perspective of the particle morphology, instead of using conventional 3D reconstruction routines [3]. These image series for the small [Fig. 1 (a)-(i)] and large [Fig. 2 (a)-(i)] particles were then compared to the 3D rotation models of the octahedron and truncated octahedron (rotated in $2^{\circ}$ steps) respectively. The different projections of the model octahedron [Fig. 1 (a1), (e1) and (i1)] and the truncated octahedron [Fig. 2 (a1), (d1) and (i1)], along with the corresponding projection axes, are shown for comparison. For example, Fig. 1(a1) is the projection image of the model octahedron representing the particle in Fig. 1(a). The 3D nanoparticle morphological observations are consistent with the nucleation and growth models [4] of the ferrite particles in plasma. These models predict that the critical nucleus shape (corresponding to the small particles) is a perfect octahedron while the growth forms (corresponding to the large particles) are truncated octahedral in shape.

References

[1] R. Swaminathan, M.E. McHenry, S. Calvin, M. Sorescu and L. Diamandescu, International Conference on Ferrites-9 Proceedings, American Ceramic Society (2004)

[2] R. Swaminathan, M.E. McHenry, P.Poddar and H.Srikanth, Journal of Applied Physics (2005)

[3] K.L.Keptra et.al, Lunar and Planetary Science XXXIV (2003)

[4] R. Swaminathan, M.A. Willard and M.E. McHenry, Acta Materialia, submitted for publication

[5] Financial support from the Institute of Complex Engineered Systems, Carnegie Mellon and Magnetics Inc. is gratefully acknowledged. 

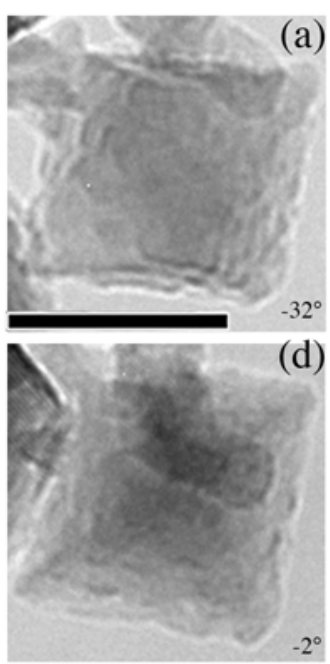

(g)

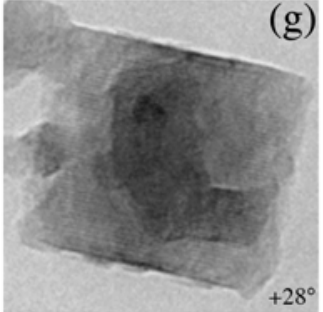

(a)

(d)
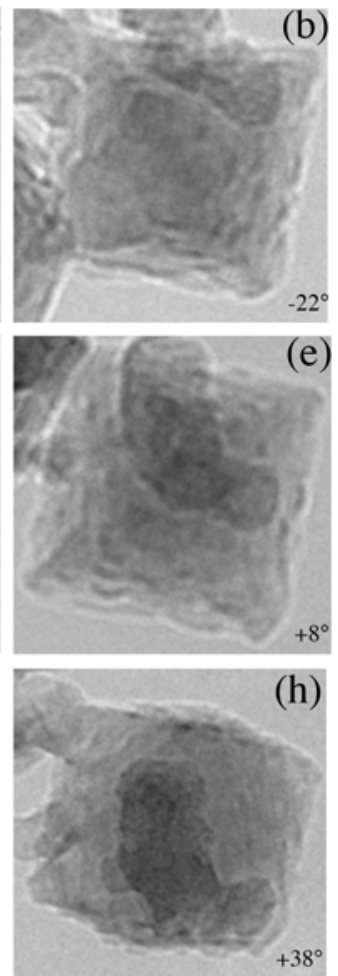

(b)
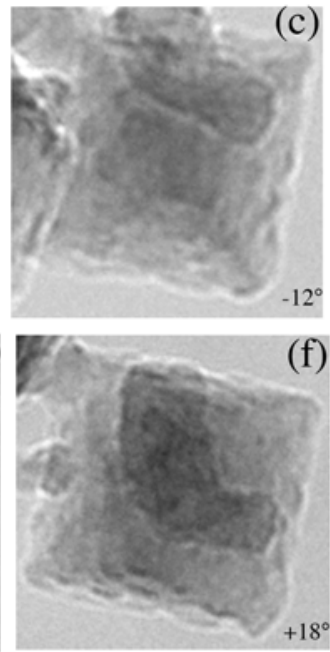

(h)

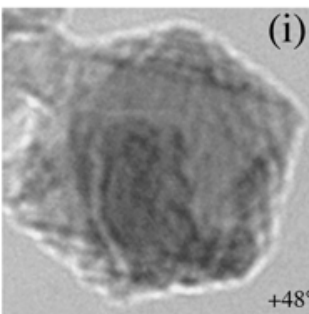

(c)

(f)
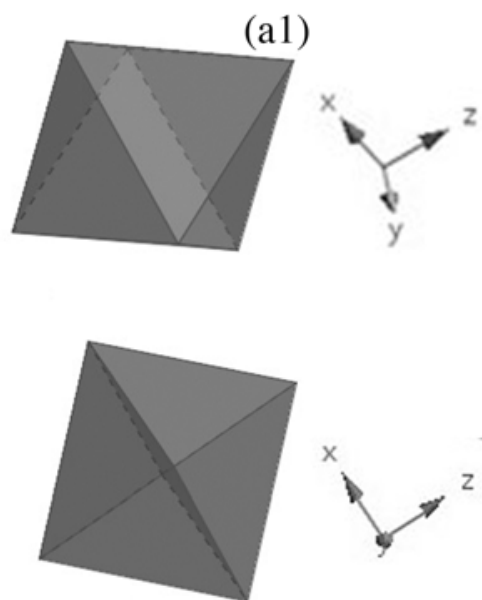

(e1)

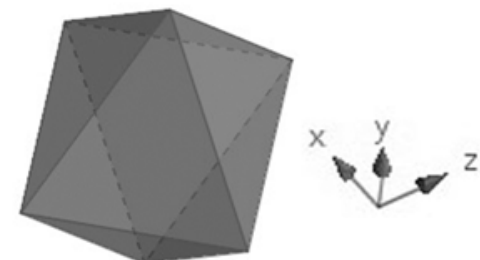

(i1)

FIG. 1. 2D projected morphologies [(a)-(i)] at different tilt angles for small octahedral nanoparticle. (a1), (e1) and (i1) are different projections of model octahedron. Scale bar on (a) is $20 \mathrm{~nm}$
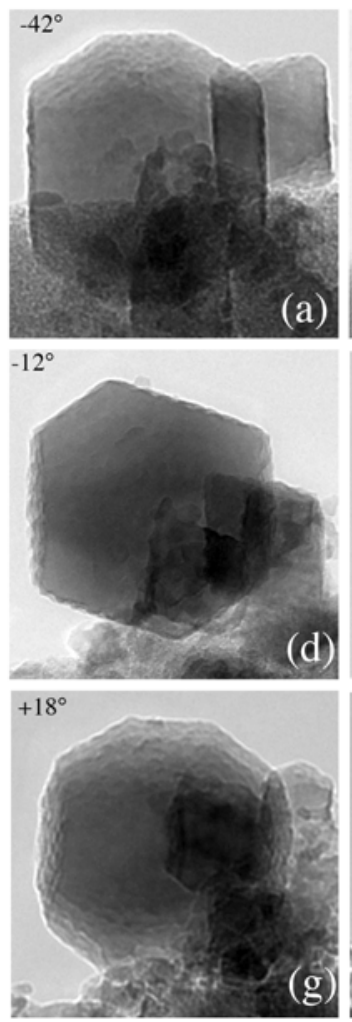
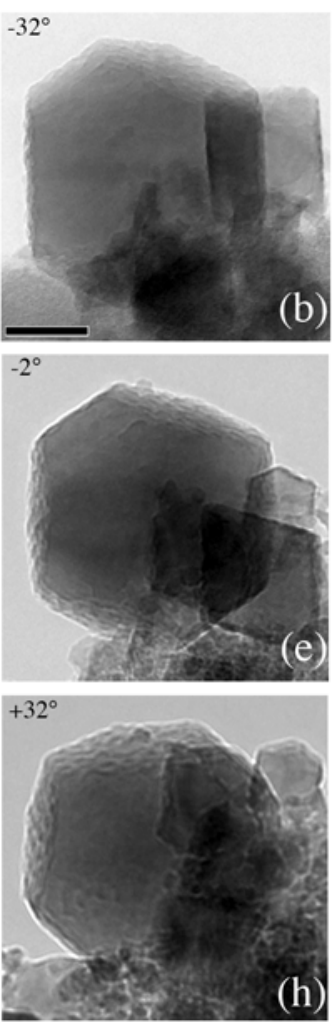

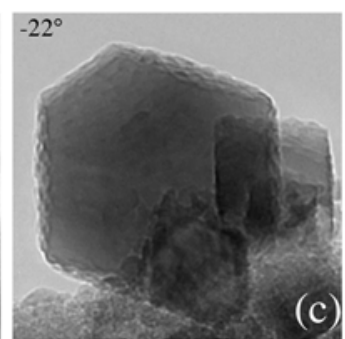

(a1)
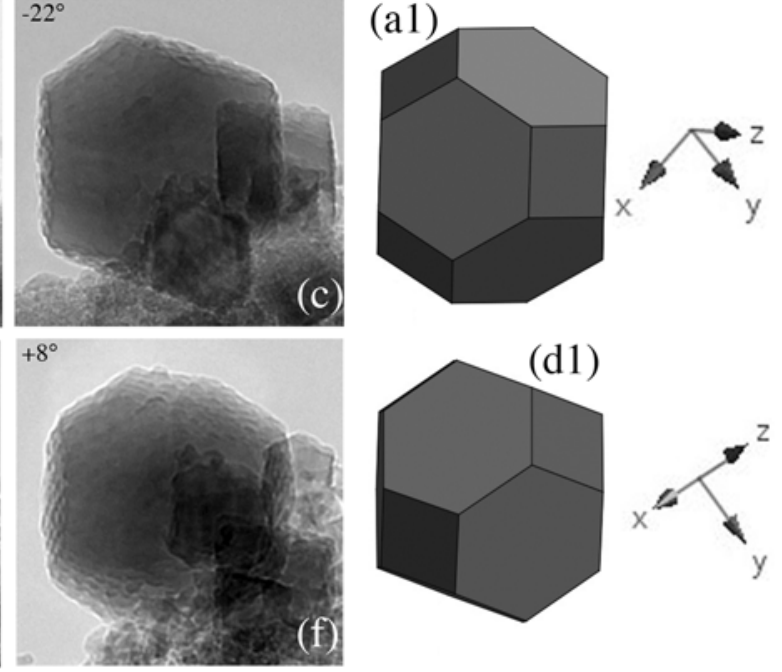

(i1)
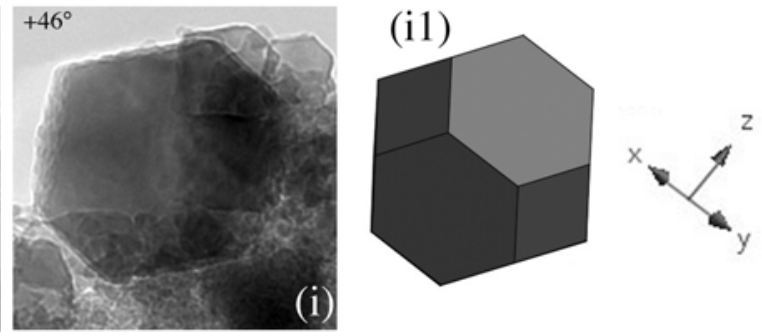

FIG. 2. Series of 2D projected morphologies [(a)-(i)] for a large truncated octahedral nanoparticle. (a1), (d1) and (i1) are different projections of model truncated octahedron. Scale bar on (b) is $20 \mathrm{~nm}$ 